Cambouis, la revue des sciences sociales aux mains sales

\title{
Négocier son entrée dans l'Armée suisse
}

\section{Stéphanie Monay}

Centre de recherche sur l'action politique, Université de Lausanne.

stephanie.monay@unil.ch

stephanie.monay@unil.ch

Résumé: Si l'institution militaire reste considérée comme un terrain fermé et opaque, elle n'est cependant pas inaccessible au chercheur ou à la chercheuse civil-e. Elle implique cependant une réflexion en amont sur les enjeux spécifiques qui conditionnent l'accès au terrain, ainsi qu'une adaptation permanente pour s'y maintenir. Dans le cadre d'une thèse portant sur la socialisation sexuée des femmes militaires engagées volontairement dans l'Armée suisse, cet article revient de manière concrète sur les différentes stratégies et pratiques de négociation mises en place afin d'accéder aux casernes et aux enquêtées. Il met également en lumière les différents obstacles et résistances rencontrés, ainsi que les concessions qui ont été nécessaires à la réalisation de la recherche. L'« art de convaincre » n'étant pas indépendant de la personne qui tente de l'appliquer, les tactiques ont dû prendre en compte ses caractéristiques sociales, à savoir une jeune femme accédant à un bastion encore fortement masculin. Cela a impliqué notamment la construction d'une crédibilité scientifique et militaire suffisante afin d'être prise au sérieux, tout en sachant paraître suffisamment « inoffensive » pour l'institution.

Date de publication : 30/06/2021

armée, femmes militaires, institution militaire, sociologie militaire, accès au terrain, négociation, méthodologie

Dossier: Pratiques et politiques de la négociation pour accéder et se maintenir sur un terrain d'enquête

Comment citer : $10.52983 /$ crev.vi0.83

Licence: Cambouis publie ses contenus selon les termes de la Licence Creative Commons Attribution - Pas d'Utilisation Commerciale - Pas de Modification 4.0 International. Les auteurices gardent leurs droits de propriété intellectuelle pleine et entière sur leurs articles. 


\section{Négocier son entrée dans l'Armée suisse}

\section{Stéphanie Monay}

Centre de recherche sur l'action politique, Université de Lausanne.

stephanie.monay@unil.ch

L'armée véhicule la réputation d'être une « grande muette » en raison d'une « culture de la restriction de l'information » (Deschaux-Beaume, 2011, p. 1) où l'accès aux documents et aux individu-e-s semble laborieux (Pajon, 2005). Cette réputation laisse donc entendre que s'y intéresser promet de s'attaquer à une " enquête au sein d'un "milieu difficile" »(Cohen, 1999, p. 17). Avant le début des années 2000, les armées restent encore peu investiguées (Bessin, 2002), notamment par les sociologues civil-e-s. Elles sont ainsi restées longtemps le quasi-monopole de la sociologie militaire, discipline composée majoritairement de chercheurs hommes passés « par les armes » (Kaya, 2013, p. 16), des sociologues insiders (Martin \& Pajon, 2015). Plus généralement, les sciences politiques et sociales ne s'intéressent encore que timidement aux terrains de la défense en comparaison à d'autres institutions étatiques. Cependant, depuis quelques années, en France notamment, l'armée fait l'objet de recherches de plus en plus poussées, réalisées notamment par des femmes civiles (Bardiès, 2017 ; Coton, 2017 ; Deschaux-Beaume, 2011 ; Paya y Pastor \& Djebbi, 2012 ; Teboul, 2017).

Dans le cadre de ma recherche de doctorat, je m'intéresse à la socialisation sexuée des quelques 1000 femmes engagées volontairement au sein de l'Armée suisse (environ $0,7 \%$ de l'effectif en 2017), contexte où le service militaire demeure obligatoire pour les citoyens tandis que les citoyennes peuvent s'engager sur un mode volontaire. Pour réaliser des entretiens de type récit de vie, il me fallait prendre contact avec des femmes militaires afin d'explorer leur socialisation sexuée antérieure à l'engagement et leurs expériences militaires actuelles. Je souhaitais également parfaire mon approche par des observations au sein de diverses casernes ${ }^{1}$, afin d'abord de m'imprégner d'un milieu qui m'était alors parfaitement inconnu, mais aussi pour dépasser un discours souvent conventionnel et très positif sur le vécu des femmes militaires suisses (Monay, 2015) et pour observer les enquêtées en situation, et plus particulièrement en situation d'interaction ${ }^{2}$. Dès lors, après consultation de la littérature scientifique, plusieurs obstacles s'annonçaient : 1, entrer dans un « univers bureaucratique particulièrement réticent à livrer des informations sur son mode de fonctionnement » (Bruneteaux, 1995, p. 110), 2, ce d'autant plus lorsqu'on y est extérieure car civile et, 3 , que l'on ne correspond pas vraiment au profil-type des individu-e-s inséré-e-s dans l'institution, à savoir une femme face à un univers fortement monosexué au masculin. À cela s'ajoutait encore le fait que, 4, l'objet de mon intérêt, les femmes militaires, pouvait potentiellement être perçu comme une menace pour l'image de l'institution militaire suisse, du fait que l'enquête débutait lors d'une phase de visibilisation - médiatique et scientifique - des violences sexistes et sexuelles vécues par les militaires féminines dans le cadre des armées occidentales, notamment en France ${ }^{3}$. Ainsi, dès le départ s'est imposée la question suivante : comment dépasser, contourner ou du moins atténuer ces différents obstacles annoncés?

J'aborderai quelques techniques d'entrée mises en place pour accéder à ce terrain et la manière dont j'ai présenté mon enquête afin qu'elle soit perçue comme inoffensive et en adéquation avec le cadre de raisonnement de
Que l'on peut considérer comme autant de sous-cultures institutionnelles militaires (Thiéblemont, 1999 ; Cohen, 2008).

2 Dans la perspective de Candace West et Don H. Zimmerman pour qui « le genre lui-même est constitué dans les interactions », celui-ci structurant en retour celles-ci, produisant ainsi de la différence sexuée (West \& Zimmerman, 2009). Ce qui impose ainsi la méthode de l'ethnographie (Avanza, Fillieule \& Masclet, 2015).

3 Principalement à travers la publication en 2014 de l'enquête journalistique de Leila Minano et Julia Pascual, La Guerre invisible : révélations sur les violences sexuelles dans l'armée française aux éditions Les Arènes et Causette. 
l'institution, tout en me garantissant les possibilités d'une analyse critique. Tout cela a cependant impliqué certaines concessions quant aux ambitions premières, ainsi qu'une renégociation constante à chaque nouvelle entrée au sein d'une sous-culture institutionnelle, soit les différentes casernes visitées qui m'ont réservé un accueil variable.

Au-delà des stratégies d'entrée - heureuses et parfois malheureuses, souvent corrigées - négociées avec la hiérarchie centrale (le Chef de l'Armée) et les hiérarchies décentralisées (commandant-e-s d'école et de centre de recrutement), il s'agissait encore d'avoir accès non pas seulement aux terrains d'enquête, mais à la parole des enquêté-e-s. Par rapport à cela, les caractéristiques de l'enquêtrice - a priori contraignantes pour elle (Fournier 2006) : une jeune femme perçue comme bien plus jeune que son âge -, ont dû parfois être compensées par la construction d'une crédibilité non seulement scientifique mais également quant aux normes qui prévalent dans le milieu d'enquête. Cependant, ces mêmes caractéristiques ont d'autres fois permis d'avoir accès à des informations et des discussions plus libres de l'emprise institutionnelle.

\section{Entrer par la « grande porte » : concessions et fébrilité}

Face à une institution fortement hiérarchique et certainement la plus centralisée de Suisse, j'ai estimé qu'obtenir un accès au terrain par la grande porte, c'est-à-dire par l'échelon le plus haut de l'institution, amènerait moins de résistances que d'approcher des casernes une à une, impliquant le bon vouloir d'une hiérarchie localisée. L'enjeu était également de pouvoir varier mes terrains d'enquête selon des logiques de recherche et non pas selon les contraintes du terrain. Il me fallait pour ce faire des intermédiaires afin de me dénicher des allié-e-s institutionnel-le-s pertinent-e-s. Cet impératif fut certainement moins compliqué dans le cadre d'une armée de milice que dans un contexte d'armée professionnelle, surtout pour une femme sans aucun lien avec l'Armée suisse : j'ai eu la chance qu'un premier assistant appartenant à mon institut avait effectué un parcours militaire poussé, ainsi que des recherches sur ce terrain. Il a ainsi pu me mettre en contact avec un officier supérieur, adjoint du Chef de l'Armée, soit un statut et un rang hiérarchique intéressants pour ma démarche. Celui-ci m’a ensuite recommandée auprès de la personne qui a été mon appui principal durant cette recherche : la femme la plus haut gradée de l'Armée suisse, la seule brigadière, également cheffe du personnel de l'armée.

J'ai rencontré la brigadière à plusieurs reprises dans son bureau à Berne. C'est une femme d'une cinquantaine d'années, droite et affirmée, qui travaille souvent en treillis militaire. Pour accéder au terrain, elle m'annonce qu'il faudra établir un dossier de présentation de la recherche indiquant mes besoins et objectifs, ainsi que les casernes et périodes pour mes observations. Ce dossier devait être ensuite validé par le Chef de l'Armée. La brigadière se montre plutôt motivée par ma recherche et prend rapidement une posture d'autorité de type « directrice de thèse » : elle est en effet titulaire d'un doctorat en chimie et connaît donc certains impératifs d'une recherche en termes de temps et de faisabilité, ce qui l'amène à s'intéresser à des aspects tels que la méthodologie, la revue de la littérature, etc. Cette première étape de réelle négociation m'amène à faire certaines concessions, comme réduire la durée de mes observations pour des questions organisationnelles internes.

Une autre concession consistait à inclure, dans le dossier de demande d'accès, le questionnaire que je souhaitais diffuser. Alors que j'estimais plus pertinent de le construire et de le diffuser après une connaissance plus pra- 
tique du terrain - permise entre autres par les observations -, j'ai dû accepter de précipiter cette étape. Dès lors, plusieurs questions se sont avérées peu pertinentes ou trop peu précises et d'autres ont manqué ${ }^{4}$. La brigadière a d'abord eu une discussion avec moi sur la base de ce questionnaire avant de le soumettre au Chef de l'Armée : pour elle, plusieurs éléments étaient à revoir. Les questions n'étaient pas perçues comme problématiques pour l'image de l'armée, mais elle estimait certaines des modalités de réponse trop ardues : «Les femmes ne vont pas prendre le temps de répondre à ça ». En effet, je souhaitais à l'origine traiter en profondeur les parcours individuels des femmes militaires (parcours militaire, professionnel, résidentiel, familial, associatif et politique) afin d'établir une analyse de type séquentiel. Cela impliquait un questionnaire conséquent avec des modalités de réponse jugées trop complexes par la brigadière. Elles ont été simplifiées et le questionnaire raccourci. Ainsi, l'objectif de la brigadière était plutôt d'assurer un taux de réponse de qualité et des questionnaires remplis dans leur intégralité. Elle a néanmoins, et la modalité d'une demande officielle aussi, eu l'effet de me faire renoncer à des questions pouvant être perçues comme menaçantes pour l'institution. La soumission du questionnaire précédant les observations, j'ai opté pour la prudence en n'insérant pas de questions sur l'existence ou l'expérience de violences sexistes et sexuelles. Celles-ci ont été traitées en profondeur dans le cadre des entretiens. Toutefois, des questions concernant la présence d'inégalités femmes-hommes au sein de l'Armée suisse étaient intégrées. Une fois mes données qualitatives obtenues (entretiens et observations), j'aurais été moins encline à cette autocensure. La démarche d'accès au terrain ayant été assez longue - j'entamais bientôt ma seconde année en thèse - j'avais d'autant plus de crainte que ma démarche s'arrête là et je souhaitais garantir mon accès aux casernes et aux femmes militaires.

Pour le dossier, j'ai opté pour plusieurs stratégies de présentation. La première consistait à rendre ma recherche peu menaçante pour l'institution en mettant la focale sur la question des raisons d'engagement des femmes militaires : «L'idée est de comprendre leur entrée volontaire dans une institution où le service militaire obligatoire et la majorité des effectifs restent masculins ». L'objet des raisons d'engagement est effectivement moins sujet à révéler d'éventuelles dysfonctions au sein de l'institution militaire, et n'insiste pas sur une posture critique de la chercheuse : une requête mobilisant des termes tels que « rapports sociaux de sexe », « discriminations » ou « harcèlement » risquait a contrario de provoquer une certaine appréhension. Il fallait toutefois me garantir la possibilité d'élargir mon questionnement, car il était effectivement plus étendu que les logiques d'engagement. Au dossier était donc ajoutée la formule suivante :

«Dans la logique d'une démarche sociologique, il s'agira également de se laisser surprendre parle terrain de recherche et ainsiélaborer de nouveaux questionnements et de nouvelles hypothèses au fil de l'enquête, selon les constats qui s'imposeront. J'en profite pour remercier le Colonel EMG $X Y$, et le Brigadier ${ }^{5} X X$ pour leur soutien et leurs conseils quant à ma démarche et ma thèse. »

L'enjeu était de faire en sorte que la question de recherche présentée ne soit pas gravée dans le marbre et éviter ainsi que ce dossier ne devienne une sorte de contrat de recherche qui encadrerait et limiterait mes analyses. Dans cet extrait du dossier de présentation, on voit également une pratique courante, très efficace dans ce milieu, celle du name dropping (placement de noms), conseillée par Deschaux-Beaume (2011, p. 9). Au niveau du choix des terrains de recherche, pour lesquels il m'a été demandé d'annoncer et dater mes intentions dans le dossier, il m'a fallu jouer sur les formules afin d'éviter des assignations à terrain définitives.
4 Le questionnaire reste tout de même conséquent et comporte plus d'une quarantaine de questions réparties en quatre sections : parcours au sein de l'Armée suisse, opinions sur la place et la condition des femmes dans l'Armée suisse, participation citoyenne et positionnement politique, caractéristiques sociales. Il a été transmis à l'ensemble des femmes militaires engagées lors de l'envoi, soit 1035 personnes. Cette méthode par le haut s'est révélée satisfaisante, avec un taux de réponse de $32 \%$.

5 Les grades et fonctions ne sont pas féminisés dans l'usage de l'Armée suisse. Si j'utilise la féminisation dans ma rédaction, mes échanges oraux et mes correspondances écrites dans le cadre du terrain s'adaptaient à cette norme afin de coller au plus près des usages du terrain. 
J'ai également insisté sur le caractère scientifique de ma démarche avec une présentation de la problématique scientifique, des hypothèses et des méthodes, le tout centré sur la question des raisons d'engagement et en mobilisant une écriture et un jargon scientifiques afin d'assoir une certaine crédibilité face à une institution d'importance en Suisse. Je n'hésitais donc pas à user des notes de bas de page faisant référence à de la littérature internationale ou de termes comme « socialisation », « entretiens de type récit de vie», « espace monosexué », « rite de passage », « division sexuelle du travail », etc.

J'ai très vite compris également, dans mes interactions avec la brigadière et mon précédant allié, qu'un « retour sur investissement » était attendu par l'institution. On m'a d'ailleurs fait sentir à plusieurs reprises que ma présence dans les casernes engendrait des difficultés organisationnelles. Je n'ai donc pas manqué d'insister sur l'apport de cette recherche pour l'institution :

«Elle lui permettra de mieux connaître qui s'intéresse à la servir sur une base volontaire et quelles sont les attentes des femmes engagées dans l'institution militaire. »

Au niveau des méthodes, le fait de proposer un questionnaire a permis de présenter un outil de recueil de données plus légitime aux yeux de l'institution que les observations, auquel on accorde plus volontiers un certain gage et une aura de scientificité ${ }^{6}$. Cela permettait également de faire miroiter des données intéressantes et plus facilement mobilisables pour et par l'institution militaire. La demande a été ensuite rapidement acceptée. Mais la négociation d'accès au terrain ne s'est pas arrêtée là.

\section{Les terrains d'enquête : renégocier à l'échelle locale}

La brigadière m'a ensuite recommandée auprès des commandements des places d'armes. Avec cet appui hiérarchique, les retours étaient rapides et positifs. Cependant, une fois sur place, l'accueil que me réservaient les hiérarchies locales était varié et mes attentes, demandes et objectifs n'étaient pas forcément compris ou jugés pertinents. On percevait en effet difficilement la pertinence d'observer le quotidien militaire. Ce n'était in fine pas si étonnant : je me suis moi-même parfois demandé l'intérêt d'observer durant plus d'une heure une section nettoyer et cirer ses bottes de combat. Dans mon optique d'observer le genre « en train de se faire », ces moments d'observation, apparemment anodins et sans intérêt, révélaient cependant « les réseaux sociaux, les normes et les règles informelles ou les stéréotypes sexués mobilisés dans l'interaction » (Buscatto, 2010, p. 120). De plus, on ne peut estimer a priori les moments d'observation qui vont se révéler « parlants » quant à une problématique spécifique. Lors de l'entretien de l'équipement personnel, il était intéressant de constater les formes de camaraderie et de solidarité entre les recrues afin de terminer cette tâche au plus vite. Celles-ci incluaient ou excluaient certaines personnes, distribuaient des rôles (l'aidé-e ou l'aidant-e), et ce notamment selon des logiques sexuées. De plus, partager ces instants
6 Comme le souligne Olivier Martin : « dans une culture où la science est centrale et représentée comme détenant la vérité sur le monde à travers des lois quantitatives, toutes les données chiffrées s'apparentent à des résultats scientifiques, donc à des vérités. Les statistiques présentent à ce point les trait du "savoir authentique" " (Martin, 1997, p. 174). 
monotones du quotidien militaire me permettait d'instaurer un rapport de confiance avec les enquêté-e-s et de multiplier les opportunités d'échanges (Stevanovic, 2014).

Les raisons de ma présence n'ont cependant pas toujours convaincu mes interlocuteur-trice-s, comme l'illustre ma première entrée dans une école de recrues.

\section{lère journée d'observation dans une caserne : une école de recrues d'infanterie}

Le commandant d'école, son adjoint et un major s'asseyent face à moi et reviennent sur ma lettre de demande d'accès à leur caserne, transmise par l'intermédiaire de la brigadière. Ils me demandent « des précisions », notamment ma « question de recherche précise ». Je suis quelque peu déstabilisée par le ton sec et froid de leur accueil et je commence à expliciter ces éléments : je m'intéresse aux profils des femmes dans l'armée, à déterminer ce qui les amène à faire le choix d'un service militaire volontaire et comment leur service se passe concrètement. Je fais certainement ici l'erreur de rester dans un registre très vulgarisé. Ils n'ont pas l'air convaincu. Ils me demandent alors quelles sont mes hypothèses : je leur donne des éléments tirés de la littérature comme l'héritage familial, les motivations professionnelles, etc. Une fois de plus, ils n'ont pas l'air satisfait, en particulier le commandant d'école qui me rétorque que je n'ai « pas de questions très précises ». Il enchaine : «Alors on a essayé de vous préparer un programme pour que vous rencontriez toutes les femmes sur la place d'armes. Il y en a dix, mais on ne peut pas vous garantir qu'on ait le temps de toutes vous les présenter ». Le major m'accompagne toute la journée : «On l'a libéré pour vous », insiste le commandant d'un ton sec, faisant comprendre assez clairement que ma présence engendre des coûts organisationnels et dérange le quotidien de la caserne. Je les remercie et la discussion se termine là-dessus. Nous sortons avec le major pour commencer les observations.

Plus tard dans la journée, je me rends avec le major à la cantine pour discuter de mes prochaines visites. Il me propose quelques arrangements par rapport à ma lettre : mes prochaines observations seront allouées aux entretiens avec les femmes militaires de la caserne, sur place. J'avais pourtant insisté sur mon souhait de les réaliser hors du service. Je sens cependant que je ne peux pas refuser cet arrangement: « On n'aura pas grand-chose d'autres à vous montrer $»$, dit-il.

Lors de ces observations, le major restait présent durant mes interactions avec les femmes militaires, ce qui était souvent gênant car cela soumettait les enquêtées au regard hiérarchique et on pouvait m'identifier comme étant du côté de l'institution, de sa hiérarchie, « l'œil du patron » (Fournier, 1996, p. 116). De même, lors des entretiens sur place, il n'était pas rare d'être dérangées ou entourées d'autres militaires, ce qui ne garantissait pas un entretien mené hors de la surveillance institutionnelle.

La mésaventure aurait pu s'arrêter là. Mais la hiérarchie de cette place d'armes a ensuite contacté la brigadière afin de se plaindre de mon manque de « questions précises », ce qui m'a valu une réprimande lors d'une rencontre quelques jours après. J'ai pu néanmoins défendre ma posture et ma recherche, et il n'y a pas eu d'incidences néfastes pour la suite. Les expériences suivantes avec cette hiérarchie locale ont cependant encore été difficiles.

Face à cette forme de résistance, qui découle en partie du fait que «les agents ne maîtrisent pas [forcément] les frontières de la pertinence sociologique » (Bruneteaux, 1995, p. 117), impliquant « une justification permanente » (Horvat, 2013, p. 64) de ma présence sur le terrain, j’ai par la suite revu la 
présentation de ma recherche. J'ai en effet renoncé à l'exposer de manière vulgarisée pour mobiliser du jargon plus sociologique afin de me construire une réelle crédibilité de type « scientifique ». Cela n'a au final pas été vraiment utile par la suite : dans les autres casernes non-rattachées à l'infanterie, l'accueil a été beaucoup plus bienveillant. J'ai parfois pu me déplacer librement sur les places d'armes, sans aucun-e chaperon-ne, et accéder à des discours plus libérés de l'emprise institutionnelle.

Ces modalités variées de prise en charge de la chercheuse ont permis de préciser les « sous-cultures institutionnelles » (Biland, 2011) présentes au sein de l'Armée suisse relatives aux types d'arme (infanterie, sanitaire, sauvetage, etc.) qui revendiquent chacune leurs spécificités (symboles, langage, chants, savoir-faire, rapports à l'ordre et à la discipline, rites, etc.) et une certaine autonomie. L'infanterie, où l'accueil a été le plus difficile et empreint de résistances, est une arme plus tardivement « féminisée », proche du combat et d'une plus stricte militarité ${ }^{7}$ (Thiéblemont, 1999 ; Cohen, 2008, p. 19-28). Elle a également une posture plus austère face aux femmes militaires, comme une faible place laissée à la féminité et une séparation plus stricte et contrôlée des baraquements et entre les sexes. En effet, dans les trois casernes visitées reliées à cette arme, la séparation des sexes était plus rigoureuse que dans celles de l'aviation ou du domaine sanitaire par exemple, ouvertes aux femmes depuis plus longtemps et où elles sont plus nombreuses sur une même place d'arme ${ }^{8}$. J'analyse cette posture peu encline vis-à-vis de ma présence comme un indicateur supplémentaire d'une sous-culture institutionnelle plus éprise de militarité, d'entre-soi et d'esprit de corps.

\section{Se construire une crédibilité tout en paraissant inoffensive}

Les conclusions quej'ai pu tirer de l'expérience relatée ci-dessus correspondent à l'expérience de Bruneautaux quant à sa thèse sur les forces de maintien de l'ordre, où ce type de terrain suppose « deux ensembles de "tactiques" d'entrisme - au sens de Michel de Certeau : d'abord une construction de respectabilité ; ensuite une gestion des résistances » (Bruneautaux, 1995, p. 111). Les caractéristiques sociales de l'enquêtrice jouent alors de tout leur poids quant à cette construction de « respectabilité » auquel je préfère ici le terme de « crédibilité ». À l'image des enquêtées, qui expérimentent du fait de leur appartenance sexuée un certain « déficit de crédibilité » (Zolesio, 2011) aux yeux de leurs camarades d'armes, de l'institution et de la société (voir Monay, 2017 et 2018), j'ai également dû « faire mes preuves » (Zolesio, 2011) par la démonstration de mes connaissances du monde militaire suisse (règlements, grades, jargons, etc.), ce qui était particulièrement attendu de la part des plus hautes hiérarchies des casernes. Si dans le cadre de ma première visite au sein de l'infanterie, on a mis en doute cette crédibilité, je me suis ensuite appliquée à la construire et à la renforcer. Christelle Coton, qui a travaillé sur les officiers de l'armée française, a pu se donner une respectabilité dans ce milieu grâce à sa filiation militaire par son père, lui-même officier (Coton, 2017).

Pour ma part, j'ai essayé de montrer à mes interlocuteur-trice-s que je détenais un certain bagage militaire et que je pouvais me soumettre au formalisme interne : j'ai tenté d'apprendre les us et normes du milieu, comme par exemple la reconnaissance rapide des insignes portés par les militaires (grades, arme, fonction, etc.). Saluer une personne par son grade en observant son insigne, surtout lorsque l'on est civile, a été particulièrement bien vu sur le terrain. Dans la même logique, dans le contexte d'un pays qui se caractérise par l'existence et la pratique de quatre langues nationales, maîtriser
7 «Ensemble des marqueurs (professionnels, juridiques, sociaux, idéologiques, culturels, corporels) attachés à la fonction militaire qui est autant une profession qu'un mode d'être. » (Paveau, 2000, p. 46).

8 Cela peut être également lié aux caractéristiques sociales des commandant-e-s des casernes. En effet, l'une des plus strictes quant aux marques de féminité (comme le maquillage) était cependant l'une des plus féminisées : le commandement était assuré par une femme pour qui il était important de garantir une égalité de traitement entre femmes et hommes et de gommer au mieux les traits distinctifs entre les sexes. Pour l'analyse des rapports à la féminité des femmes militaires dans l'Armée suisse, voir Monay, 2018. 
la traduction français-allemand des fonctions et des grades m'a également permis de gagner une certaine légitimité, certain-e-s cadres peinant parfois elles et eux-mêmes à trouver rapidement leurs termes dans une autre langue. S'imprégner du lexique indigène et des codes sociaux non formalisés dans les documents, soit « aménager ses pratiques le plus possible dans le sens d'un mimétisme pondéré » (Bruneteaux, 1995, p. 113), a été un moyen de paraître quelque peu avertie et de faire oublier mon statut d'intruse.

La crédibilité scientifique devait également être tangible pour les hiérarchies des places d'armes, avec la promesse que la recherche apporte quelque chose à l'institution, comme on peut le comprendre dans les propos d'un commandant d'école :

«Il y a un regain d'intérêt pour les femmes dans l'armée, là on a bien trois ou quatre recherches là-dessus [des travaux de diplôme essentiellement, principalement de la formation secondaire voire tertiaire (travail de maturité gymnasiale, séminaire de bachelor universitaire, diplôme d'école professionnelle)], et des demandes pour venir. Mais moi si ce n'est pas sérieux je dis non, il faut que ça amène une plus-value et j'ai lu quelques travaux et ça n'apportait rien. Votre recherche, c'est bon, c'est l'université, c'est scientifique, alors là je veux bien. »

Ce discours montre le poids de la réputation universitaire par rapport à d'autres écoles et niveaux de formation, mais aussi le fait que des demandes adressées directement aux hiérarchies décentralisées pouvaient être refusées. La démarche consistant à passer par les hautes sphères hiérarchiques centralisées semble donc bien celle adéquate pour ce type de terrain.

Mes caractéristiques sociales ont également pu devenir un atout sur le terrain militaire afin d'accéder à une parole libérée du formalisme militaire et du devoir de réserve. En effet, le fait d'être une femme faisant bien plus jeune que son âge m'a permis d'être perçue comme inoffensive.

\section{« Vous faites un exposé pour quelle école?»}

Je participe à un cycle de recrutement mixte dans le centre romand. J'ai la possibilité d'assister à toutes les séances hormis les visites médicales et psychologiques, et je peux prendre part à tous les entretiens individuels d'affectation des candidates au service militaire, ce qui me permet de récolter de nombreuses informations. Durant ces deux journées, les cadres sont assez décontracté-e-s et me parlent volontiers d'aspects très personnels, comme leur parcours militaire ou encore leurs opinions - souvent critiques - sur la place des femmes dans l'Armée suisse. Par exemple, l'une des sergentes me confie que, selon elle, il y a des candidates qui réalisent un service militaire pour des " raisons sexuelles », à savoir disposer d'un grand nombre d'hommes avec lesquels coucher durant le service. Ainsi, souvent, les propos tenus n'étaient pas contenus par la volonté de donner une image progressiste de l'institution militaire ou par la crainte d'avoir face à soi une personne « hostile » à l'armée (Fournier, 1996). Le deuxième jour, le commandant du centre me présente son prédécesseur, un colonel EMG récemment parti à la retraite. Celui-ci me serre la main et me salue d'une manière enjouée. Il me parle des femmes militaires en usant d'un ton quelque peu paternaliste. Il me demande alors : « Vous faites un exposé pour quelle école ?». Un peu étonnée du terme employé, je lui réponds que je suis à l'université : «Ah! Vous êtes en bachelor? » Je lui précise que non, qu'il s'agit d'une thèse de doctorat. Le colonel se redresse, visiblement interloqué et me dit, avec un ton cette fois plus sérieux : « Ah mais vous savez, il y a des choses ici qui sont confidentielles! »a.

a. Il ne ma cependant jamais été précisé quels éléments devaient rester confidentiels lors de mes observations. Dès lors, jai pu profiter de ce flou. Cependant, certains documents internes sont restés inaccessibles car jugés confidentiels. 
Les mêmes caractéristiques sociales peuvent ainsi jouer tour à tour en faveur ou en défaveur de la chercheuse. L’impératif ici est de repérer rapidement les attentes, les représentations voire les stéréotypes des enquêté-e-s et de tenter de s'y adapter en conséquence, tout en ayant en amont anticipé les comportements et élaboré certaines tactiques. Ainsi, s'il fallait parfois démontrer mes compétences académiques et/ou ma connaissance du terrain d'enquête pour être jugée crédible, il était parfois plus efficace de faire preuve de naïveté : les militaires - en particulier les femmes militaires - étaient souvent ravi-e-s de m'expliquer les ficelles du métier, le jargon, les traditions, les pratiques militaires. Sur ce point, mon expérience rejoint celle explicitée par Deschaux-Beaume : le fait d'être une enquêtrice dans un milieu presque exclusivement masculin pouvait être un avantage dans le sens où « il est possible de jouer sur le registre des questions "naïves" qui permettent d'obtenir beaucoup d'informations sur les pratiques et les représentations des acteurs militaires » (Deschaux-Beaume, 2011, p. 13). De même, mon apparente jeunesse me permettait d'aller au-delà des effets de censure, car elle me donnait une image inoffensive, loin de celle de «l'intellectuel-le critique », comme l'a aussi expérimenté Geneviève Pruvost sur le terrain de la police (Pruvost, 2014, p. 20).

Pour l'accès à la parole de mes principales enquêtées, à savoir les femmes militaires, mes caractéristiques sociales jouaient également en ma faveur. Nous avions en effet une proximité en termes d'âge - du moins c'est ce qu'elles percevaient - et elles me considéraient généralement comme une femme qui portait de l'intérêt au monde militaire et avec laquelle elles pouvaient partager cette « particularité » que souligne leur forte minorité au sein de l'armée. En effet, lors des entretiens, les enquêtées supposaient chez moi un fort attachement à l'institution militaire et me prêtaient ainsi « des croyances communes » aux leurs (Roulleau-Berger, 2004, p. 226). Cependant, si j'ai pu collecter de nombreux contacts lors de mes observations, les entretiens ne se sont que rarement concrétisés (moins de la moitié) : c'est auprès des femmes militaires que j'ai pu rencontrer de manière répétée sur le terrain que mes demandes d'entretien ont été les plus fructueuses. Le contact répété avec les enquêtées, formel et informel comme lors de repas ou de sorties, a donc permis de créer progressivement une confiance suffisante, ou du moins de « normaliser» ma présence et mes questionnements, pour qu'elles acceptent l'exercice de l'entretien.

Anticiper les accueils variables faits à ma recherche sur le terrain militaire permettait ainsi de multiplier les tactiques possibles et d'opter, le moment venu, pour la plus pertinente, même si cela ne garantissait pas toujours un accès et un accueil optimaux. Des indicateurs aussi impossibles à dissimuler que mon genre et mon âge pouvaient jouer à mon avantage ou être quelque peu « corrigés » par la démonstration de compétences et du sérieux de ma démarche. Mais cela ne suffisait bien entendu pas toujours à retourner des situations : là où j'ai essuyé le plus de résistances, il demeure difficile à affirmer que telle ou telle tactique aurait permis de les dépasser. Cela ne représente pas un simple échec de négociation pour l'accès et le maintien dans le terrain : certes, celui-ci peut aller jusqu'à vous exclure, mais cette exclusion même, et les diverses formes de résistances, informent également sur les logiques qui structurent les différents milieux que nous investiguons. Ainsi, revenir et réfléchir sur les diverses étapes de la négociation a pu m’apprendre beaucoup quant aux différentes armes qui composent l'armée : l'attitude face à une chercheuse (et ses caractéristiques sociales) ainsi que les modalités de sa prise en charge sont autant d'indicateurs des spécificités des sous-cultures institutionnelles étudiées, en particulier leur position quant à la présence de femmes militaires dans leurs rangs. 
L'armée n'est pas si muette, et son silence en dit parfois beaucoup. L'enjeu principal était ici de trouver un équilibre et de savoir s'adapter entre une posture sérieuse, crédible et une image inoffensive. Doublement « outsider » car civile et femme, mes enquêté-e-s pouvaient supposer que je me plaçais dans une posture d' " hostilité de principe » vis-à-vis de l'armée (Martin \& Pajon, 2015), impliquant méfiance, censure, résistance de leur part. Cependant, faire la démonstration de ma connaissance des us et coutumes, du jargon, des logiques et codes militaires, de même que poser des questions et montrer un intérêt sincère pour les vécus des militaires rencontré-e-s, a permis d'amoindrir peu à peu la défiance à mon égard. En effet, par ce comportement je faisais la démonstration du fait que j'accordais au monde militaire une certaine légitimité, du moins suffisamment pour réaliser une recherche à son propos. Comme le conclut Geneviève Pruvost sur le terrain policier - qui partage de nombreuses caractéristiques avec le milieu militaire -, « la clef d'entrée dans un tel milieu, c'est en fait la démonstration de son caractère inoffensif » (Pruvost, 2014, p. 22), auquel le genre féminin et la jeunesse peuvent contribuer. 


\section{Bibliographie}

Avanza Martina, Fillieule Olivier, Masclet Camille, 2015, «Ethnographie du genre. Petit détour par les cuisines et suggestions d'accompagnement », SociologieS, http://journals.openedition.org/ sociologies/5071.

BARDIÈs Laure, 2017, « La sociologie française et la chose militaire. Une tradition antipolitique », Revue française de science politique, vol. 67, $\mathrm{n}^{\circ} 5$, p. 879-898.

Bessin Marc, 2002, Autopsie du service militaire 1965-2001, Paris, Autrement, 205 p.

BILAND Émilie, 2011, « Les cultures d'institution », dans Jacques LAGROYE, Michel Offerlé (dir.), Sociologie de l'institution, Paris, Belin, p. 177192.

BRUneteaux Pierre, 1995, « Manœuvres scientifiques en terrain militaire », Genèses, $\mathrm{n}^{\circ}$ 19, p. 108-121.

Buscatto Marie, 2010, La Fabrique de l'ethnographe. Dans les rouages du travail organisé, Toulouse, Octarès, 168 p.

CoHen Samy, 1999, «Enquête au sein d'un "milieu difficile" : les responsables de la politique étrangère et de la défense », dans Samy Cohen (dir.), L'art d'interviewer les dirigeants, Paris, PUF, p. 17-50.

Cohen Samy, 2008, « Le pouvoir politique et l'armée », Pouvoirs, ${ }^{\circ} 125$, p. 19-28.

Coтon Christel, 2017, Officiers. Des classes en lutte sous l'uniforme, Marseille, Agone, 288 p.

DeschauX-Beaume Delphine, 2011, « Enquêter en milieu militaire. Stratégie qualitative et conduite d'entretiens dans le domaine de la défense », Res Militaris, vol. $1, \mathrm{n}^{\circ} 2$.

FOURNIER Pierre, 1996, « Des observations sous surveillance », Genèses, n 24, p. 103-119.

FouRNiER Pierre, 2006, « Le sexe et l'âge de l'ethnographe : éclairants pour l'enquêté, contraignants pour l'enquêteur », ethnographiques.org, $\mathrm{n}^{\mathrm{o}} 11$, https://www.ethnographiques.org/2006/Fournier.

Horvat Nicolas, 2013, « Le regard critique en situation d'immersion », Socio-anthropologie, $\mathrm{n}^{\circ} 27$, p. 59-69.

KAYA Sümbül, 2013, La production militaire du citoyen. Sociologie politique de la conscription en Turquie, Thèse de doctorat en Science politique, Université Paris I/Panthéon Sorbonne.

MARTIN Clément, PAJON Christophe, 2015, « La sociologie militaire par les personnels de la défense : une sociologie d'insiders? », Les Champs de Mars, $\mathrm{n}^{\circ}$ 27, p. 23-30.

MARTIN Olivier, 1997, « "Les statistiques parlent d'elles-mêmes ». Regards sur la construction sociale des statistiques », dans Club MerleauPonty, La pensée confisquée, Paris, La Découverte, p. 173-191.

MonAY Stéphanie, 2015, « Les femmes dans l'Armée suisse : un discours entre égalité et stéréotypes de genre », dans Claude WEBER (dir.), Les femmes militaires, Rennes, PUR, p. 207-218.

MonaY Stéphanie, 2017, « La constance du "naturel". Processus de sélection et d'affectation des femmes volontaires dans l'Armée suisse », Sextant, $\mathrm{n}^{0} 34$, p. 35-44.

MonAY Stéphanie, 2018, « Femmes dans l'armée Suisse. Une socialisation à la domination masculine », Sociétés contemporaines, $\mathrm{n}^{\circ} 112$, p. 45-71. 
PAJON Christophe, 2005, «Le sociologue enrégimenté. Méthodes et techniques d'enquête en milieu militaire », dans François Gresle (dir.), Sociologie du milieu militaire. Les conséquences de la professionnalisation sur les armées et l'identité militaire, Paris, L'Harmattan, p. 45-56.

PAVEAu Marie-Anne, 2000, « Les frontières discursives de la militarité », Langage et société, $\mathrm{n}^{\circ}$ 94, p. 45-74.

PAYA y PAStor Alicia, DJEbBi Sihem, 2012, « Vers la structuration d'un champ de la sociologie militaire. Débats théoriques, méthodologiques et stratégiques », Lettre de l'ISREM, n 9, https:// www.defense.gouv.fr/english/irsem/publications/lettre-de-l-irsem/ les-lettres-de-l-irsem-2012-2013/2012-lettre-de-l-irsem/lettre-de-lirsem-n-9-2012/releve-strategique/vers-la-structuration-d-un-champde-la-sociologie-militaire.-debats-theoriques-methodologiques-etstrategiques.

Pruvost Geneviève, 2014, « Ni policier ni homme : une sociologue enquête sur la féminisation de la police », dans Anne MonJARET, Catherine Pugeault (dir.), Le sexe de l'enquête : approches sociologiques et anthropologiques, Lyon, ENS, http://books.openedition.org/ enseditions/3979.

ROULLEAU-BERGER Laurence, 2004, « Voir, "savoir-être avec", rendre public : pour une ethnographie de la reconnaissance », Cahiers internationaux de sociologie, $\mathrm{n}^{\circ} 117$, p. 261-283.

SteVANOVIC Jasmina, 2014, « Les femmes officiers de la marine marchande. Rapports sociaux de sexe, intimité, pouvoir », dans Anne MonjARET, Catherine Pugeault (dir.), Le sexe de l'enquête : approches sociologiques et anthropologiques, Lyon, ENS, https://books. openedition.org/enseditions/3974.

Teboul Jeanne, 2017, Corps combattant. La production du soldat, Paris, Maison des Sciences de l'Homme.

Thiéblemont André (dir.), 1999, Cultures et logiques militaires, Paris, PUF.

West Candace, Zimmerman Don H., 2009, «Faire le genre », Nouvelles Questions Féministes, vol. 28, n ${ }^{\circ}$ 3, p. 34-61.

ZoLesio Emmanuelle, 2011, «De la nécessité pour les femmes chirurgiens de "faire leurs preuves". Surinvestissement professionnel et grossesse », Questions Vives, vol. 8, no 15, p. 61-75. 